\title{
Formulas of Integration of Permissivity and of Directed Molecular Targeting In Oncogenesis
}

\author{
Lawrence M Agius* \\ Department of pathology, University Of Malta Medical School, Malta,
}

Submission: December 15, 2017; Published: January 05, 2018

"Correspondence Address: Lawrence M Agius, Department of pathology, University of Malta Medical School, Mater Dei Hospital Msida, TALQROQQ, Malta, Europe, Tel: 356-21451752; Email: lawrence.agius@um.edu.mt

\begin{abstract}
Dimensions of non-resolution or paradoxically of resolved integrative action allow for a dualized context of constitutive receptor activity and of permissiveness in the creation of the malignant phenotype of cancer cells. The proposition for such permissiveness is compounded by resolved end-result in oncogenesis that is projected in terms of aberrant over-activity of tyrosine kinases that include dimensions of over-activity in the face of non-resolved dynamics of spread and metastasis. It is within such dimensional context that the oncogenesis is portrayed as established pathways within a permissive context for further change progression. Growth factors and transcription factors allow for such permissiveness to integrate with the directed targeting of molecules in terms of protein-protein interactivity and tyrosine kinase formulations.
\end{abstract}

\section{Introduction}

Intracellular and receptor tyrosine kinases constitute attractive targets for interfering with progression of carcinogenesis. Strong evidence highlights the association of autophagy with tyrosine kinase receptor signaling [1]. It appears that both broad groups of tyrosine kinases constitute an integral complex based especially on protein-protein interaction consequent to phosphorylation/dephosphorylation of the tyrosine kinase domain. It would further appear that the progression of a given cancer lesion is susceptible to manipulation of kinase activity in terms of such pathway effects through the pathway supportive indices as projected by Src activation on the one hand and of regulatory subunits such as adaptor proteins or phosphatases. Aryl hydrocarbon receptor may act as a protein adaptor to recruit kinases by bypassing Epidermal Growth Factor and may drive resistance to tyrosine kinase inhibitors [2]. The phosphorylation by-pathways are integral intermediate regulation steps in the activation and inactivation of pathways that create constitutive signaling cascades and the provision of intra-nuclear targeting as exemplified by Abl. MYC-driven, BRAFi-resistant cells are hypersensitive to the inhibition of MYC synthetic lethal partners in melanoma, including SRC family and c-KIT tyrosine kinases [3].

\section{Cell Phenotype}

The cell phenotype is an expressed domain activated formula in the definitive transformation of events in terms of a complex integration of intracellular and receptor tyrosine kinase formulation. Protein phosphorylation is strictly regulated by protein kinases and protein phosphatases [4]. The significance in provision of activated/de-activated motif formulation is further compounded by tyrosine kinase binding in the presence of scaffold proteins in inducing progression of pathway kinetics. Functionally activating HER2 mutations may drive and maintain cancers in a manner analogous to HER2 gene amplification and HER2 mutations may confer sensitivity to HER2-directed drugs [5].

\section{Interactivity}

In triple negative breast cancer, emerging therapeutic targets include PARP1, receptor and non-receptor tyrosine kinases, immune-checkpoints, androgen receptor and epigenetic proteins [6]. Simple interactivity between phosphorylated tyrosine kinase and protein intermediates is further projected in terms of absent ligand binding domains that induce constitutive activation of a number of tyrosine kinase domain-containing receptors. Tyrosine kinases are an especially important target as they modulate growth factor signaling [7]. In such measure the significant overlap of protein moieties allow replacement of entity protein molecules as exemplified by Jak1 and Jak2.

The projection pathways are often implicated in the regulated expression of transcription factors that, within the nucleus, control the active participation of gene expression. Sorafenib, a small inhibitor of tyrosine protein kinase, is currently the standard chemotherapy drug for the treatment of advanced 
hepatocellular carcinoma [8,9]. Monoclonal antibodies, small molecule tyrosine kinase inhibitors and epigenetic drugs are being currently tested in clinical trials [8]. Growth factors such as Her2/Neu, Epidermal Growth Factor, Vascular Endothelial Growth Factor, allow for the integration of signaling cascades within projected formulations of response to cell phenotypes, as well proposed by cytoskeletal response and motility/ invasion, and also especially by the increased proliferative rate as a universal response of transformed cancer cells. It is further toward the shape and instability as induced by malignant transformation that there evolve dimensions of proliferative rate that centrally express the abnormal and constitutive activity of tyrosine kinases found linked to receptors. The use of targeted small molecule inhibitors is revolutionizing the treatment of non-small cell lung cancer [10].

\section{Integrated Tyrosine Kinase Action}

A key to integrated tyrosine kinase action as a strictly oncogenic mechanism allows for the identification of system parallels that call into operative performance the constitutive activity of tyrosine kinase receptors. Recent advances in Next Generation Sequencing have allowed significant insights into the genomic characteristics of human cancers [11]. In such manner, the resolving integration that is afforded by intracellular tyrosine kinases is involved in transfer dynamics of such constitutive activation of the receptor tyrosine kinases per se. It is common for cancer cells to exhibit deregulation of several tyrosine kinases simultaneously, and multikinase TKIs [tyrosine kinase inhibitors] therefore offer greater advantages over single-target TKIs [12]. Simplified outlines of pathway progression induce a progression processing that requires substrate binding within the added contexts of scaffold protein modalities.

Promotional interactivities permit a resolution of integration in activities such as would create essential constitutive parameters of acquisition in terms of dual and multiple impact in multi-formulations as exemplified by systems for further transformation. Inhibition or elimination of phospholipase D activity involves proliferative signaling by receptor tyrosine kinases and G protein-coupled receptors [13]. Transformed gene expression profiles are significant in carcinogenesis in terms of contextual parameters of cooperative systems of receptor and intracellular tyrosine kinase activities.

\section{Protein-Protein Interaction}

Protein-protein interactivities are further promoted inter-play in conjunction with constitutive receptivity that lends an additional dimension to the progression kinetics of carcinogenetic pathways. Autonomous and constitutively active receptors integrate with formulas of cell responsiveness in the creation of transfer dynamics beyond the simple concept of permissive instability of genetic origin. Tyrosine kinase inhibitors combined with hepatic resection have a role in improving the outcome of patients with gastrointestinal stromal tumors that metastasise to the liver [14]. It is clear that the indicated permissiveness of genetic instability allows for the creation of tyrosine kinase responses in their own right. Tyrosine kinase FYN-a member of the SRC family of kinases is associated with mediating mlitogenesis and regulates cell cycle entry, growth, and proliferation, including in thyroid carcinoma [15].

Such dual reciprocity is well-exemplified by the relative bidirectional dynamics between intracellular tyrosine kinases and growth factors such as those displayed by Src and Epidermal Growth Factor activity. Such dual reciprocity allows for the Src to influence and also be influenced by stimulatory actions of Epidermal Growth Factor or by the expression of Vascular Endothelial Growth factor in the induced angiogenesis that accompanies proliferation of the transformed cancer cells. The tumor-suppressor protein Mig-6 directly targets and inhibits the human ErbB receptor tyrosine kinases ErbB1 and ErbB2, even through these two kinases are separately involved in the pathogenesis of different types of human tumors [16].

\section{Constitutive Receptivity}

ZAP70 is the only gene that is differentially expressed in CD5-high or CD5-low normal lymphocytes confirming the key role of Zap-70 tyrosine kinase in BCR signaling alterations in B-Chronic lymphocytic leukaemia [17]. The mechanics of constitutive receptor activations in their formulated identity profiles are parameter for an integrating formula that signifies further progression of the transformation step in oncogenesis. The ubiquitous nature of alternative splicing events in RON [Recepteur d'origins nantais] receptor is suggestive of the need for isoform specific approaches to functional analysis and therapeutic targeting of RON [18]. Protein-protein interactions allow for dysregulation of site-promoted and site-regulated localization of substrate. Also, the folding of the Src molecules is further propounded by systems of accessibility of binding sites as exemplified by Src and also Abl.

\section{Outline Dynamics}

The outline dynamics of integrated interactivities are projected as common central pathways of progression in oncogenesis that reflect the representation of the kinetic binding sequences as further proposed by systems of non-resolution of permissive contexts of progressive transformation.

The dynamics of protein-protein interactions are resulting dimensions of phosphorylation and dephosphorylation in the additional context of a dualized receptivity and of intracellular tyrosine kinase over-response. Both erlotinib alone and in combination with bevacizumab inhibits tumor growth in the gefitinib-sensitive lung cancer mice model, with decreased vascular perfusion, decreased micro vessel density and increased tumor apoptosis [19]. Amplified activation pathways as proposed for point mutations and of translocation events between different chromosomes allows for the creation of dimensional resolution as integrated interactivities. Such 
proposed context allows for the formulation of predisposed or facilitated outlines in pathway progression leading to a predisposed and oncogenically transformed cell phenotype.

Several receptor tyrosine kinase ligands increase RhoAguanosine triphosphate in untransformed and transformed cell lines depending on the RTKs activating the AKT serine/threonine kinase [20]. Homology 2 and Homology 3 domains of Src are model domain activities within such formulations that identify integration in carcinogenesis. It is further projected as mirror image dimensions in terms of formulas of non-resolution on the one hand and as integral dimensions of cooperative interactivity of constitutive tyrosine kinase receptors and of intracellular tyrosine kinase formulations in induced carcinogenesis. Raf and Mek co-inhibition exhibits synergy in triple negative breast cancer models and constitutes a promising combination therapy for this aggressive breast cancer type [21].

\section{Conclusion}

Phosphorylation and dephosphorylation events emerge as terms of reference in tyrosine kinase formulations as further exemplified by dynamics of resolution of activated pathways in inducing contexts of oncogenic transformation. In such terms, overall dimensions in the provision for transformation evolve as cell responsiveness in paradoxical integral context of permissive micro-environmental conditioning of the genetic instability status of the host cells. It is beyond the considerations of constitutive activation of receptors that there emerges a dimensional integration that is largely dependent on kinetic dynamics of the tyrosine kinase phosphorylation and dephosphorylation at the cytoplasmic domains of these enzymes.

\section{References}

1. Fraser J, Cabodevilla AG, Simpson J, Gammoh N (2017) Interplay of autophagy, receptor tyrosine kinase signalling and endocytic trafficking. Essays Biochem 61(6): 597-607.

2. Ye M, Zhang Y, Gao H, Xu Y, Jing P, et al. (2017) Activation of the ary hydrocarbon receptor leads to resistant to EGFR TKIs in non-small-cell lung cancer by activating Src-mediated bypass signaling. Clin Cancer Res.

3. Singleton KR Crawford L, Tsui E, Manchester HE, Maertens Q, Liu X, et al. (2017) Melanoma therapeutic strategies that select against resistance by exploiting MYC-driven evolutionary convergence. Cell Rep 21(10): 2796-2812.

4. Otsubo K, Yoneda T, Kaneko A, Yagi S, Furukawa K, et al. (2017) Development of a substrate identification method for Human Scp1 phosphatase using phosphorylation mimic phage display Protein Pept Lett.

5. Connell CM, Doherty GJ (2017) Activating HER2 mutations as emerging targets in multiple solid Cancers ESMO Open 2(5): e000279.

6. Lee A, Djamgoz MBA (2017) Triple negative breast cancer: emerging therapeutic modalities and novel combination therapies Cancer Treat Rev 62: 110-122.
7. Prabhu VV, Devarj N (2017) Epidermal growth factor receptor tyrosine kinase: a potential target in treatment of non-small-cell lung carcinoma J Environ Pathol Toxic Oncol 36(2): 151-158.

8. Olgen S (2017) Overview on anticancer drug design and development. Curr Med Chem.

9. Zhang SS, Ni YH, Zhao CR, Qiao Z, Yu HX, et al. (2017) Capsaicin enhances the antitumor activity of sorafenib in hepatocellular carcinoma cells and mouse xenograft tutors through increased ERK signaling Acta Pharmacy Sin.

10. Lategahn J, Keul M, Rauh D (2017) Lessons to be learned: the molecular basis of kinase-targeted therapies and drug resistance in non-small cell lung cancer Angew Chem Int Ed Engl.

11. Imperial R, Toor OM, Hussain A, Subramanian J, Masood A (2017) Comprehensive pan cancer genomic analysis reveals (RTK)-RAS-RAFMEK as a key dysregulated pathway in cancer: its clinical implications Semin Cancer Biol.

12. Xie Y, Li C, Huang Y, Jia Z, Cao J (2017) A novel multikinase inhibitor R8 exhibits potent inhibition on cancer cells through both apoptosis and autophagic cell death Oncotarget 8(50): 87209-87220.

13. Roth E, Frohman MA (2017) Proliferative and metastatic roles for Phospholipase D in mouse models of cancer Adv Biol Regul pii: S22124926(17)30178-1.

14. Shi YN, Li Y, Wang YP, Wang ZH, Liang XB, et al. (2017) Gastrointestinal stromal tumor (GIST) with liver metastases: an 18-year experience from the GIST cooperation group in North China Medicine (Baltimore) 96(46): e8240.

15. Zheng J, Li H, Xu D, Zhu H (2017) Upregulation of tyrosine kinase FYN in human thyroid carcinoma: role in modulating tumor cell proliferation, invasion, and migration. Cancer Biother Radiopharm 32(9): 320-326.

16. Deng Y, Li J (2017) Rational optimization of tutor suppressor-derived peptide inhibitor selectivity between oncogene tyrosine kinases ErbB1 and ErbB2 Arch Pharm (Weinheim).

17. Giadkikh AA, Potashnikova DM, Tatarskly V Jr, Yastrebova M Khamidulline A, et al. (2017) Comparison of the mRNA expression profile of B-cell receptor components in normal CD5-high B-lymphocytes and chronic lymphocytic leukaemia: a key role of ZAP70 Cancer Med 6(12): 2984-2997.

18. Krishnaswamy S, Mohammed AK, Tripathi G, Alokail MS, Al-Daghri NM, et al. (2017) Splice variants of the extracellular region of RON receptor tyrosine kinase in lung cancer cell lines identified by PCR and sequencing. BMC Cancer 17(1): 738.

19. Chen YF, Yuan A, Cho KH, Lu YC, Kuo MY, et al. (2017) Functional evaluation of therapeutic response of HCC827 lung cancer to bevacizumab and erlotinib targeted therapy using dynamic contrastenhanced and diffusion-weighted MRI. PLoS One 12(11): e0187824.

20. Tripathi BK, Grant T, Qian X, Zhou M, Mertins P, et al. (2017) Receptor tyrsine kinase activation of RhoA is mediated by AKT phosphorylation of DLC1 J Cell Biol 216(12): 4255-4270.

21. Nagaria TS, Shi C, Leduc C, Hoskin V, Sirdar S, et al. (2017) Combined targeting of Raf and Mek synergistically tumorigenesis in triple negative breast cancer model systems. Oncotarget 8(46): 8080480819. 
Your next submission with Juniper Publishers will reach you the below assets

- Quality Editorial service

- Swift Peer Review

- Reprints availability

- E-prints Service

- Manuscript Podcast for convenient understanding

- Global attainment for your research

- Manuscript accessibility in different formats ( Pdf, E-pub, Full Text, Audio)

- Unceasing customer service

Track the below URL for one-step submission https://juniperpublishers.com/online-submission.php 